\title{
A mapping study on blood glucose recommender system for patients with gestational diabetes mellitus
}

\author{
Shuhada Mohd Rosli, Marshima Mohd Rosli , Rosmawati Nordin \\ Faculty of Computer \& Mathematical Sciences, Universiti Teknologi MARA, Shah Alam, Malaysia
}

\begin{abstract}
Article Info
Article history:

Received Apr 30, 2019

Revised Jun 7, 2019

Accepted Jul 10, 2019

\section{Keywords:}

Blood glucose

GDM

Gestational diabetes mellitus

Recommender system

Systematic mapping

ABSTRACT

Blood glucose (BG) prediction system can help gestational diabetes mellitus (GDM) patient to improve the BG control with managing their dietary intake based on healthy food. Many techniques have been developed to deal with blood glucose prediction, especially those for recommender system. In this study, we conduct a systematic mapping study to investigate recent research about BG prediction in recommender systems. This study describes an overview of research (2014-2018) about BG prediction techniques that has been used for BG recommender system. As results, 25 studies concerning $\mathrm{BG}$ prediction in recommender system were selected. We observed that although there is numerous studies published, only a few studies took serious discussion about techniques used to incorporate the BG algorithms. Our result highlighted that only one study discusses hybrid filtering technique in BG recommender system for GDM even though it has an ability to learn from experience and to improve prediction performance. We hope that this study will encourage researchers to consider not only machine learning and artificial intelligent techniques but also hybrid filtering technique for BG recommender system in the future research.
\end{abstract}

Copyright $\odot 2019$ Institute of Advanced Engineering and Science. All rights reserved.

\section{Corresponding Author:}

Marshima Mohd Rosli,

Department of Computer Science,

Faculty of Computer and Mathematical Sciences,

Universiti Teknologi MARA, Malaysia

Email: marshima@tmsk.uitm.edu.my

\section{INTRODUCTION}

Diabetes is one of the commonest chronic medical conditions, influencing around 347 million adults worldwide [1]. Diabetes management should avoid acute and long-term complications that can be responsible for premature death and disability. The influence of diabetes is developing with 347 million people at present influenced worldwide and numbers anticipated to increment to 552 million by 2030 [1]. In the UK, the cost used by National health Service (NHS) related to diabetes in 2002 was assessed to be around $£ 1.3$ billion consistently [2], with the majority of this cost emerging from the long haul difficulties coming about because of diabetes not being managed properly [3].

Gestational diabetes mellitus (GDM) is frequently described as glucose intolerance for pregnancy women. A primary concern with GDM is the patient could have perinatal complications such as cesarean delivery, birth injury, neonatal metabolic disorders and preeclampsia [4]. GDM also have an impact for long term metabolism consequences for the mother and children from the pregnancy such as increased risk of obesity [5]. Thus, pregnancy women should maintain normal BG levels to prevent adverse pregnancy outcomes and to break the cycle of transmission of metabolic disease to children [6].

GDM has health consequences for both the mother and her children not only in the short term but also in the long term [7]. Mothers with record of GDM have significantly higher risk of GDM during 
subsequent pregnancies (2), type 2 diabetes and premature cardiovascular disease. Children of GDM pregnancy have greater risk of developing obesity, diabetes, hypertension and cardiovascular disease [8].

The timeframe for interventions to avoid complications from GDM is within the third trimester of pregnancy. Pregnant women with GDM need to ensure their glycemic control is within a good range. They need to visit the health care every 2-4 weeks for GDM treatment such as food intake treated with insulin. Although these visits are similar to general antenatal visits, patients with GDM are often felt stress because they need to spend longer time due to the limited of resources at the health care [9].

Technology can be used to remotely provide health care that offers an appealing solution to this problem. Currently, a growing wide variety of research are reporting over mobile apps because of patients along different kinds concerning diabetes. Some of the research describe the controlled experiments based on mobile phone that indicate reliable results to improve the performance of self-management on diabetes [10-13]. These mobile apps stated as advantageous tool because of the tool ability of making appropriate food intakes to avoid high BG levels since postprandial glucose (PPG) responses would be of particularly significance for women with GDM.

Besides, patients with GDM are advised to monitor daily other factors that can influence their health state, for example administered insulin doses, ingested diet, exercise, or other relevant factors. This condition increases the amount of data generated in diabetes care and the patient's and physician's workload when analyzing data to take therapeutic decisions [14]. Thus, it is important to have the appropriate infrastructure that incorporates data processing algorithms.

Incomplete BG data is one of the reasons that limit the effectiveness of decision support systems in diabetes [15]. A diary management in web-based platform is developed along including records exchange and continuous BG sign technology software. Both aspects were joined to gain the fundamental statistics for utilizes of the personalized BG recommender system. Essential statistics over meals, BG measurements, then other events were amassed by the carried out web-based and continuous glucose monitoring (CGM) law technology software. These data were used to tune then evaluate the BG recommender model, who protected an algorithm because of strong coefficients tuning [16].

Despite the fact that several BG recommender algorithms have been proposed, only a small number of projects are committed to the development of complete recommender system infrastructures that incorporate BG recommender for GDM patients [17]. The development and implementation of such a system might be particularly significant for patients with GDM, especially considering the high importance of BG control for these patients during pregnancy.

As we reviewed the literature, we found several existing techniques for recommender system [18-26], but we do not know which one is the most appropriate for BG recommender system, particularly for patients with GDM. Therefore, in this study, we review research studies about BG recommender system that used several techniques to incorporate the BG recommender system and BG models for GDM. This paper presents a mapping study to investigate recent research about BG recommender system for patients with gestational diabetes mellitus. The contributions of this paper are:

a. A classification scheme categorizing the papers of BG techniques for recommender system.

b. A systematic mapping study of BG techniques for recommender system, structuring related research work by analysing 25 selected papers.

The remainder of this paper is organized as follows: Section 2 describes the systematic mapping methodology. Section 3 reports the results of the mapping study. Section 4 discusses the important findings of the mapping study. Finally, we conclude and provide recommendation in Section 5.

\section{METHODOLOGY}

A systematic mapping study is used to outline the relevant research publication in a specific research zone and arrange the essential research production in that area into defined categories [27-29]. The mapping study used high-level research questions to investigate issues that reflect the importance of the research. The research questions are formulated in such a way to allow researcher to categorize the current work, to identify future areas of research and to define the search strategy and paper selection criteria [28]. The mapping results examines high-frequencies of current research trends outlined in tables or visualized in bar plots, and these can uncover potential research regions that will be suitable for performing a systematic review [29].

A systematic mapping study normally requires less exertion and incorporates more significant articles than a systematic review because of mapping study perform general assessment (e.g., abstract, introduction and conclusion) to distinguish a specific context of each publication and develop the classification scheme. On the other hand, a systematic review implements a detailed and focused evaluation 
for only chosen empirical publication to guarantee the assessment results depend on great quality evidence. The systematic mapping process as proposed by [29] consists of five activities:

a. Definition of research question

This paper uses a narrative literature review approach to compare and summarize on the techniques of BG recommender system for GDM in the literature. This paper analysed the selected studies based on the following research questions:

RQ1: What method or techniques in BG recommender system are addressed in recent research?

RQ2: What data collection used in BG recommender system are addressed in recent research?

RQ3: What number and types of variables in BG prediction models are addressed in recent research?

b. Conduct search for primary study

1) Search strategy

The fundamental assignment to identify primary studies is to develop search string dependent on defined method, data collection, blood glucose recommender system, variables and blood glucose prediction models. We formulated the search terms through the following step [29]:

- The main terms are determined by identifying the method, data collection, blood glucose recommender system, variables and blood glucose prediction models.

- Keywords are identified in the relevant papers.

- Synonyms of the keywords are identified.

- Search strings are formulated by using Boolean operators (OR, AND) to incorporate alternative spellings, synonyms and to link the major term.

The search query was formulated as follow:

(method OR technique OR approach) AND (data collection OR data gathering) AND (Blood glucose recommender system OR BG recommender system) AND (variables OR variable OR at-tribute) AND (BG prediction models OR Blood glucose prediction models).

2) Search process

The search process included papers from vital conferences and journals regarding on research area, for example, Journal of System \& Software and Journal of Medical Internet Research. We restricted our research in computer science and software engineering domain. The search results are summarized in Table 1.

Table 1. Summary of Primary and secondary search

\begin{tabular}{clccc}
\hline No. & Database Name & No. of search result & No. of duplicate found & No. of relevant articles \\
\hline 1. & PubMed & 12 & 0 & 9 \\
2. & SpringerLink & 4 & 2 & 0 \\
3. & ACM Digital Library & 978 & 6 & 6 \\
4. & Academia & 7524 & 125 & 7 \\
5. & Goggle Scholar & 1190 & 0 & 3 \\
& Total & 9708 & 133 & 25 \\
\hline
\end{tabular}

c. Screening of papers for inclusion and exclusion

We defined the following inclusion and exclusion criteria for filtering process to retrieve the most relevant papers. The inclusion and exclusion criteria are:

- Inclusion: Peer reviewed papers that related to BG recommender system using BG prediction models.

- Exclusion: Papers describe methods or techniques for BG recommender system but not using prediction models.

We obtained a set of 9708 papers after execution of automatic search. The set was reduced to 9575 papers after screening the titles and abstracts and excluding irrelevant and duplicate papers. We carried out the screening of papers by reading the introduction and conclusion of each paper. We found that only 25 papers are relevant with this study.

d. Developing the classification scheme

We applied a key wording approach using abstract [29] to determine new classification schemes plans for the chose papers. The approach involved two part. The initial part included reading the abstract for each paper and observed potential keywords and idea to recognise context of the research. The following part was combining keywords and concepts to generate a general interpretation regarding the nature and commitment of the research. After performing this step, we chose a final set of keyword to form the categories.

e. Data extraction

In this part, we used an Excel spreadsheet to document the data and generate the results. We collected all information required to address the research questions. 


\section{RESULTS}

\subsection{Method or techniques in BG recommender system (RQ1)}

As mentioned earlier, previous study $[18,19,20,21]$ in recommender system indicate despite the fact that several BG recommender algorithms have been proposed, only a small number of projects are committed to the development of complete recommender system infrastructures and deal with chronic diseases such as GDM. In our study, the total of relevant result is 25 studies but we only obtained 20 studies that satisfied our inclusion criteria. The outcome from a systematic mapping process is illustrated in Figure 1 that presents the distribution of studies on techniques for BG recommender system.

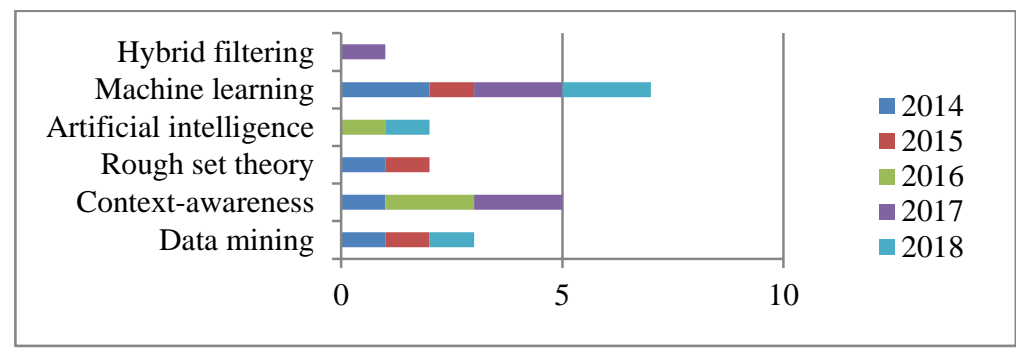

Figure 1. Distribution of studies on techniques for BG recommender system

As can be seen, the number of studies for techniques in BG recommender system for machine learning and context awareness are increased from 2014 to 2018. There is a high number of studies on the machine learning techniques whereas only one study contributed on the hybrid filtering technique for BG recommender system in 2017. The research community also has contributed an average amount of studies on the artificial intelligent techniques, rough set theory techniques and data mining techniques.

\subsection{Data collection used in BG recommender system (RQ2)}

As mentioned earlier, a variety of terminology was used to discuss data collection used in BG recommender system. The collection of data can be previous blood glucose values, carbohydrate ingestion, insulin intake and so on [18]. Table 2 show the relevant papers according to data collection methods that used to manage the $\mathrm{BG}$ prediction models in $\mathrm{BG}$ recommender system.

Table 2. Distribution of paper on data collection method

\begin{tabular}{ccc}
\hline Database & Data collection method & No of paper \\
\hline \multirow{2}{*}{ PubMed } & Dynamic coefficients tuning & 1 \\
& Context fusioning & 1 \\
ACM Digital Library & Cloud based architecture & 1 \\
& Imputation & 1 \\
\hline
\end{tabular}

As can be seen in Table 2, only 5 out of 25 researches that have mentioned about data collection method used in BG recommender system which are dynamic coefficients tuning, context fusioning, cloud based architecture, imputation and feed forward artificial neural networks (ANNs). It shared the same number of research which is one of each used different data collection method to manage the BG prediction models.

\subsection{Number and types of variables in BG prediction models (RQ3) \\ 3.3.1. Participants' characteristics}

There are six major characteristics of the participants in the BG prediction models $[18,30]$ such as age (years), prepregnancy BMI, glycated hemoglobin (HbA), Gestational age (weeks), blood pressure, blood pressure (BP) systolic, blood pressure diastolic etcetera. Figure 2 shows three studies from the relevant papers that indicated the participants characteristics in the BG prediction models.

As can be seen in Figure 2, we found a study used all the participant characteristic as their variables in BG prediction models in 2018. We found a study that used medical records, gene expression data and medical image data as their variables but only mentioned age as their participants characteristics [30]. We 
also found another study that used age, BMI data, BP systolic and BP diastolic as their measurement data to predict BP with recurrent neural network [31].

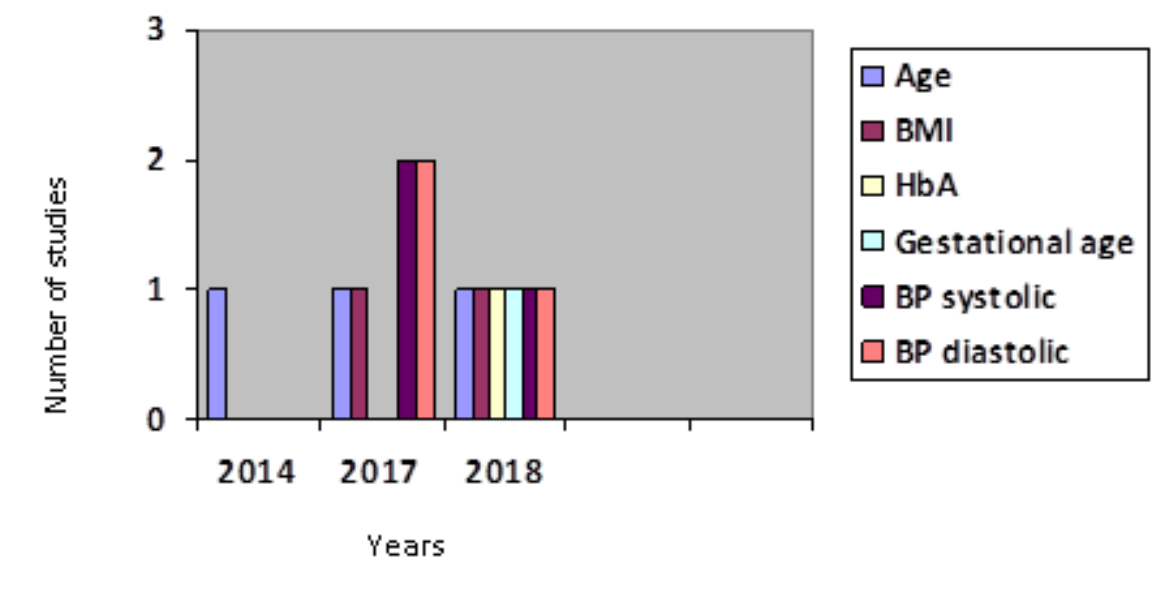

Figure 2. Frequency of participant characteristic used in BG prediction models

\subsubsection{Characteristics of meals}

The energy content of patients with GDM meals was considerably lower than the normal diabetics because they consumed lower amounts of carbohydrates and fats in their meals. Besides, fasting and postprandial BG levels were significantly higher in patients with GDM than in those in the normal diabetes, whereas the actual rise in BG level after meals did not vary significantly in these groups owing to lower average carbohydrate consumption in the GDM [11]. Table 3 illustrates the distribution of relevant papers for 10 characteristics of meals.

Table 3. Distribution of papers on characteristics of meals

\begin{tabular}{|c|c|c|}
\hline No & Characteristic & No of paper \\
\hline 1 & Fasting BG & 6 \\
\hline 2 & BG level 60 minute after the meal & 2 \\
\hline 3 & Area under the postprandial BG curve 60 minutes after the meal & 2 \\
\hline 4 & Area under the postprandial BG curve 120 minutes after the meal & 2 \\
\hline 5 & BG rise 1 hour after meal & 2 \\
\hline 6 & Postprandial peak BG & 1 \\
\hline 7 & Time to peak BG & 1 \\
\hline 8 & Carbohydrates per meal & 4 \\
\hline 9 & Proteins per meal & 3 \\
\hline \multirow[t]{2}{*}{10} & Energy per meal & 2 \\
\hline & Total & 25 \\
\hline
\end{tabular}

As we can see in Table 3, 6 papers indicated that GDM patients used Fasting BG as the characteristics of meals to manage their blood glucose level. We found 4 papers indicated that GDM patients used carbohydrates per meal to control their blood glucose level. In addition, we also found 2 papers mentioned about postprandial peak BG and time to peak BG as the characteristics of meals for GDM patients in managing their blood glucose level.

\section{DISCUSSIONS}

This paper was designed to determine the status of recent research on BG recommender system for GDM. We performed a mapping study to identify the BG techniques, data collection method in BG recommender system and number and types of variables in BG prediction model. We found 25 papers that have mentioned the BG techniques, the data collection method and the types of variables used in the BG recommender system. 
In reviewing the literature, we found many papers discussed on machine learning technique and artificial intelligence technique. A study conducted by Gema indicated that the machine learning technique is used in a decision-making algorithm with the ability to integrate food choices based on PPG response models [15]. This will enable the app to give personalized advice concerning each upcoming meal in order to achieve desired BG levels. However, there is the potential for biases due to inaccurate reports because it use small sample size.

Artificial intelligence techniques are being increasingly established, as it is suitable for use in clinical daily practice [19]. Consequently, these techniques provide powerful tools for improving patients' quality of life. Furthermore, the availability of genetic data, such as that provided by metabolomics analysis, has also empowered the application of artificial intelligence techniques to personalization of diabetes management. But, the increasingly frequent use of health apps and the potential use of tools based on artificial intelligence by insurance companies could lead to discrimination or the exclusion (or both) of some citizens from health services.

One interesting finding is only 1 paper was found discussed on hybrid filtering technique. Hybrid filtering technique is a combination of content-based filtering technique [22] and collaborative filtering technique [23]. The hybrid filtering technique consists of both content-based filtering and collaborative filtering techniques advantages [32]. It improves the performance by overcoming the drawbacks of traditional recommendation systems. A study conducted by Contreas et al. indicated that this technique can identify patient profiles and generate tailored prediction models for future BG values [25]. The method uses a classifier to identify patient profiles by analyzing their BG dynamics and administered insulin dosages. Another study conducted by Roshini et al. also indicated that hybrid recommendation engine is a competent system as recommender for users, whereas the other recommender algorithms are quite slow with inaccuracies [33]. Therefore, this indicates that further research is needed to explore on the hybrid filtering technique in $\mathrm{BG}$ prediction models for $\mathrm{BG}$ recommender system.

\section{CONCLUSION}

The purpose of this study is to review research papers about BG prediction in recommender system. Currently, there are exist so many technique for recommender system but we do not know which one is the most appropriate for BG recommender system. Besides, until recently this has not been the case in BG recommender system for patients with GDM since most of the studies discussed about BG recommender for diabetes.

This study has revealed that few research considered hybrid filtering technique in BG recommender system even though it have an ability to learn from experience and can improve prediction performance. This finding indicates that more effort needs to be made by the research community to explore the strength and capabilities of hybrid filtering techniques to improve the accuracy of blood glucose prediction for recommender system. Moreover, a greater focus on hybrid filtering technique could produce interesting findings that account more for BG recommender system for patients with GDM.

We are currently working on constructing algorithm for food dietary intake prediction based on blood glucose level using hybrid filtering technique and, in the future, to develop web application for food intake prediction for GDM. It is hoped that this will encourage the research community to consider hybrid filtering technique in BG recommender system to improve BG control in patients with GDM.

\section{ACKNOWLEDGMENTS}

The authors would like to thank the Universiti Teknologi Mara for their financial support to this project under BESTARI Grant No. 600-IRMI/PERDANA 5/3 BESTARI (105/2018).

\section{REFERENCES}

[1] Pal K, E. S. "Computer-based diabetes self-management interventions for adults with type 2 diabetes mellitus (Review)". The Cochrane Collaboration, 1-74. 2013.

[2] M. Alotaibi and I. Choudhury, "A social robotics children diabetes management and educational system for Saudi Arabia: System architecture," 2015 Second International Conference on Computer Science, Computer Engineering, and Social Media (CSCESM), Lodz, 2015, pp. 170-174.

[3] Jannoo, Z., Yap, B. W., Lazim, A. M., \& Hassali, M. A. "Examining diabetes distress, medication adherence, diabetes self-care activities, diabetes-specific quality of life and health-related quality of life among type 2 diabetes mellitus patients". Journal of Clinical and Translational Endocrinology, 9, 48-54. 2017.

[4] Metzger BE, L. L. "Hyperglycemia and adverse pregnancy outcomes. HAPO Study Cooperative Research Group". The New England Journal of Medicine. 2008. 
[5] Silverman BL, R. T. "Long-term effects of the intrauterine environment". The Northwestern University Diabetes in Pregnancy Center. Diabetes Care, 142-149. 1998.

[6] El Hajj N, S. E. "Epigenetics and life-long consequences of an adverse nutritional and diabetic intrauterine environment". Reproduction, 111-120. 2014.

[7] Wei-Wei Zhu, H.-X. Y.-M.-L.-R.-H. "Evaluation of the Valu eof Fasting Plasma Glucose in the First Prenatal Visit to Diagnose Gestational Diabetes Mellitus in China". Epidemiology/Health Services Research, 586-590. 2013.

[8] S. Mutalib, S. Abdul-Rahman and A. Mohamed, "Mining Frequent Patterns for Genetic Variants Associated to Diabetes," 2014 25th International Workshop on Database and Expert Systems Applications, Munich, 2014, pp. 28-32.

[9] McCauley, K. "Psychological issues for women diagnosed with gestational diabetes mellitus". ResearchGate. 2014.

[10] Bonoto BC, d. A. "Efficacy of mobile apps to support the care of patients with diabetes mellitus: a systematic review and meta-analysis of randomized controlled trials". JMIR Mhealth Uhealth. 2017.

[11] Liang X, W. Q. "Effect of mobile phone intervention for diabetes on glycaemic control: a meta-analysis". Diabet Med, 455-463. 2011.

[12] Holtz B, L. C. "Diabetes management via mobile phones: a systematic review". Telemed J E Health, 175-184. 2012.

[13] Association, A. D. "Standards of medical care in diabetes". Clin Diabetes, 3-12. 2016.

[14] Balaji Bhavadharini, M. M. "Use of capillary blood glucose for screening for gestational diabetes mellitus in resource-constrained settings". Springer. 2018.

[15] Gema Garc'ia-S'aez, J. M.-S. "Mealtime blood glucose classifier based on Fuzzy Logic for the DIABTel telemedicine system". Link Springer, 1-10. 2009.

[16] Saila B. Koivusalo, M. M.-L. "Gestational Diabetes Mellitus Can Be Prevented by Lifestyle Intervention: The Finnish Gestational Diabetes Prevention Study (RADIEL)”. Crossmark, 24-30. 2016.

[17] Oviedo S, V. J. "A review of personalized blood glucose prediction strategies for T1DM patients". Int J Numer Method Biomed Eng. 2017.

[18] Evgenii Pustozerov, P. P. "Development and Evaluation of a Mobile Personalized Blood Glucose Prediction System for Patients With Gestational Diabetes Mellitus”. JMIR Mhealth Uhealth. 2018.

[19] Ivan Contreras, J. V. "Artificial Intelligence for Diabetes Management and Decision Support: Literature Review". Journal of Medical Internet Research. 2018.

[20] N. L. Adam, M. A. Zulkafli, S. C. Soh and N. A. M. Kamal, "Preliminary study on educational recommender system," 2017 IEEE Conference on e-Learning, e-Management and e-Services (IC3e), Miri, 2017, pp. 97-101.

[21] Mohamed Mahtar SN, Khairudin N, Masrom S, Jaafar SN, Abdul Rahim SKN, Azizan A. "Reliable hadith recommender system". Advanced Science Letters. Oct 1;22(10):2936-2940. 2016.

[22] Adam NL, Zulkafli MA, Soh SC, Kamal NAM, Bakar NA. "Personalized recommender system for calculus using content-based filtering approach”. International Journal of Engineering and Technology(UAE). Jan 1;7(3):110-113. 2018.

[23] N. N. Yusof, A. Mohamed and S. Abdul-Rahman, "Reviewing Personalizing Filtering Approaches in Web," 2014 4th International Conference on Artificial Intelligence with Applications in Engineering and Technology, Kota Kinabalu, 2014, pp. 63-68.

[24] Rahman Ali, J. H. "H2RM: A Hybrid Rough Set Reasoning Model for Prediction and Management of Diabetes Mellitus". Sensors (Basel). 2015.

[25] I. Contreras, J. Vehi, R. Visentin and M. Vettoretti, "A Hybrid Clustering Prediction for Type 1 Diabetes Aid: Towards Decision Support Systems Based upon Scenario Profile Analysis," 2017 IEEE/ACM International Conference on Connected Health: Applications, Systems and Engineering Technologies (CHASE), Philadelphia, PA, 2017, pp. 64-69.

[26] Md Saiful Islam, M. M.-E.-A. "A Systematic Review on Healthcare Analytics: Application and Theoretical Perspective of Data Mining". PubMed. 2018.

[27] J. Bailey, D. Budgen, M. Turner, B. Kitchenham, P. Brereton and S. Linkman, "Evidence relating to Object-Oriented software design: A survey," First International Symposium on Empirical Software Engineering and Measurement (ESEM 2007), Madrid, 2007, pp. 482-484.

[28] D. Budgen, M. T. "Using mapping studies in software engineering". Proc. of PPIG, 195-204. 2008.

[29] K. Petersen, R. S. "Systematic mapping studies in software engineering". Int. Conf. on Evaluation and Assessment in Software Engineering. 2008.

[30] Milan Vukićević, S. R. "Cloud Based Metalearning System for Predictive Modeling of Biomedical Data”. Scientific World Journal. 2014

[31] Xiaohan Li, S. W. "Blood Pressure Prediction via Recurrent Models with Contextual Layer". $W W W$ '17 Proceedings of the 26th International Conference on World Wide Web, 685-693. 2017.

[32] Kaustubh Kulkarni, K. W. "A Study Of Recommender Systems With Hybrid Collaborative Filtering". International Research Journal of Engineering and Technology (IRJET), 2216-2219. 2016.

[33] Roshni Padate, J. K. "Hybrid Recommendation System Using Clustering and Collaborative Filtering". International Journal on Recent and Innovation Trends in Computing and Communication, 305-310. 2017. 